\title{
Comparison of Kurtosis and Fourth Power Detectors with Applications to IR-UWB OOK Systems
}

\author{
Javad Taghipour, Vahid Tabataba Vakili, Dariush Abbasi-Moghadam \\ Department of Telecommunications, School of Electrical Engineering, Iran University of \\ Science and Technology (IUST), Tehran, Iran \\ Email: jtaghipour@elec.iust.ac.ir, vakily@iust.ac.ir, abbasi@ee.iust.ac.ir
}

Received October 22, 2011; revised December 5, 2011; accepted December 17, 2011

\begin{abstract}
On-off keying (OOK) is one of the modulation schemes for non-coherent impulse radio Ultra-wideband systems. In this paper, the utilization of the kurtosis detector (KD) and fourth power detector (FD) receivers for OOK signaling is introduced. We investigate the effect of integration interval and the optimum threshold on the performance of energy detector (ED), KD and FD receivers. The semi analytic expression of BER is obtained by using generalized extreme value distribution function for KD and FD receivers. From performance point of view, the simulation results show that FD receiver outperforms $\mathrm{KD}$ and $\mathrm{ED}$ receivers. In contrast, the sensitivity to the optimum threshold is greatly reduced in $\mathrm{KD}$ receiver compared to $\mathrm{ED}$ and FD receivers.
\end{abstract}

Keywords: Ultra-Wideband (UWB); Non-Coherent Receiver; OOK; Energy Detector; Kurtosis Detector; Fourth Power Detector

\section{Introduction}

Impulse Radio Ultra-wideband (IR-UWB) systems are based on the transmission of pulses with very short duration $[1,2]$. Coherent and non-coherent receivers are commonly used in IR-UWB systems. The non-coherent receivers have low complexity implementation and are used in low cost applications. In this paper, non-coherent receivers are investigated for IR-UWB on-off keying (OOK) scheme.

Energy detector (ED) is one of the non-coherent receivers for the IR-UWB signal reception [3]. ED receivers usually are used for pulse position modulation (PPM) and OOK signaling in IR-UWB systems. The decision mechanism in PPM is made by sign detector, and in OOK scheme, by comparing the output of energy integrator with a threshold value. Threshold value in OOK signaling is investigated in [4-6]. Comparing the various transmission schemes in [3], it is confirmed that OOK modulation with ED outperforms the PPM scheme. So in this paper, OOK signaling is used for IR-UWB non-coherent system.

Kurtosis detector (KD) [7] and fourth power detector (FD) [8] are recently proposed non-coherent detectors to enhance the performance of energy detector. Simulation results in [7] and [8] show that the KD and FD receivers has a lower bit error rate than ED receiver for a IR-UWB PPM system using IEEE 802.15 CM1 channel model.
In this paper, we propose utilizing the KD and FD receivers for IR-UWB OOK signalling scheme. The approximation of optimum threshold value for symbol decision and the semi analytic BER expression are calculated from GEV distribution function for IR-UWB OOK scheme. We show that, FD receiver outperforms KD and ED receivers, and the KD receiver outperforms in high integration intervals compare to ED receiver. We also show that, KD receiver does not require optimizing integration interval, and the KD receiver has a very low sensitivity to the optimum threshold value variations compare to ED receiver.

The rest of the paper is organized as follows. In Section 2, the system model of IR-UWB OOK is presented. Sections 3 and 4 describe the conventional ED structure and the proposed $\mathrm{KD}$ and $\mathrm{FD}$ receivers' structures for OOK scheme, respectively, for the detection of IR-UWB signals. The performance evaluation and the results are discussed in Section 5. Finally, concluding remarks are presented in Section 6.

\section{System Model}

The transmitted signal in OOK scheme can be expressed as follows

$$
s(t)=\sum_{i=-\infty}^{\infty} b_{i} \sqrt{E_{w}} w\left(t-i T_{b}\right),
$$

where $w(t)$ is the UWB pulse, $E_{w}$ is the energy of $w(t), T_{b}$ 
is the symbol time and $b_{i}\{0,1\}$ is the binary information bits.

Signal $s(t)$ propagates through a multipath channel with impulse response

$$
h(t)=\sum_{j=1}^{L} \alpha_{j} \delta\left(t-\tau_{j}\right),
$$

where $L$ is the number of multipath components, $\alpha_{k}$ and $\tau_{k}$ are the gain and delay associated with the $k$ th multipath component according to IEEE802.15.4 channel model [9], and $\delta($.$) is the Dirac delta function. Then the received$ signal can be expressed as

$$
r(t)=\sqrt{E_{w}} \sum_{i=-\infty}^{\infty} b_{i} g\left(t-i T_{b}\right)+n(t),
$$

where $n(t)$ is the white Gaussian noise with power spectral density $N_{0} / 2$, and $g(t)=w(t) * h(t)$ is the channel response to $w(t)$.

\section{Energy Detector}

An energy detector employs a square device, an energy integrator and a threshold decision mechanism which are shown in Figure 1. The decision variable in ED is obtained as follows

$$
z_{\mathrm{ED}}=\int_{0}^{T_{i}} r^{2}(t) \mathrm{d} t
$$

where $T_{i}$ is the integration interval and $r(t)$ is the received signal passing through a band pass filter.

In OOK scheme, the demodulation stage has two hypotheses

$$
\begin{cases}H_{0}: z_{\mathrm{ED}}=\int_{0}^{T_{i}} n^{2}(t) \mathrm{d} t & \text { (bit } 0) \\ H_{1}: z_{\mathrm{ED}}=\int_{0}^{T_{i}}(g(t)+n(t))^{2} \mathrm{~d} t & \text { (bit } 1)\end{cases}
$$

where $g(t)$ and $n(t)$ are the received desired signal and noise respectively. The symbol decision in receiver is made by comparing $z_{\mathrm{ED}}$ with a threshold value $T h$. If the received signal energy is lower than a threshold value, the detector decides that the transmission bit is 0 . If the received signal energy is larger than a threshold value, the detector decides that the transmission bit is 1 .

$$
\begin{cases}\text { if } & z_{\mathrm{ED}}<T h_{o p t}: H_{0} \\ \text { if } & z_{\mathrm{ED}} \geq T h_{o p t}: H_{1}\end{cases}
$$

Hypotheses 0 and 1 have the probability density functions (PDF) $p_{0}(x)$ and $p_{1}(x)$, respectively. The optimum threshold value $T h_{\text {opt }}$ is obtained by the solution of $p_{0}(x)$ $=p_{1}(x)$. The PDFs of $p_{0}(x)$ and $p_{1}(x)$ are shown to be

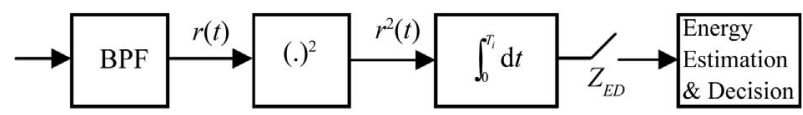

Figure 1. Energy detector (ED). central and non-central chi square distribution $\left(X^{2}\right)$ respectively [4]:

$$
\left\{\begin{array}{l}
p_{0}(x)=\frac{1}{N_{0} \Gamma(M)}\left(\frac{x}{N_{0}}\right)^{M-1} \exp \left(-\frac{x}{N_{0}}\right) \\
p_{1}(x)=\frac{1}{N_{0}}\left(\frac{x}{E_{w}}\right)^{\frac{M-1}{2}} \exp \left(-\frac{x+E_{w}}{N_{0}}\right) I_{M-1}\left(2 \frac{\sqrt{x E_{w}}}{N_{0}}\right)
\end{array}\right.
$$

where $M=B T_{i}, \Gamma($.$) denote Euler function, B$ is the signal bandwidth and $I_{n}$ is the $n t h$ Bessel function of the first kind.

\section{Proposed Kurtosis Detector and Fourth Power Detector for OOK Scheme}

In this section we propose two non-coherent receivers for IR-UWB OOK signalling scheme, by using the fourth order statistics of received signal.

\subsection{Kurtosis Detector}

The Kurtosis for random variable $x$ is defined as

$$
\operatorname{kurt}(x)=E\left\{x^{4}\right\}-3\left[E\left\{x^{2}\right\}\right]^{2},
$$

where $E\{\}$ denotes the expected value of the variable. If $x$ is a Gaussian random variable, its kurtosis is zero. If $x$ has a subgaussian distribution, it means that the distribution of $x$ has flatness and shorter tails relative to Gaussian distribution, its kurtosis has a negative value. If $x$ has a supergaussian distribution, it means that the distribution of $\mathrm{x}$ has peakedness and longer tails relative to the Gaussian distribution, its kurtosis has a positive value. In impulse radio UWB, the received signal has a supergaussian distribution in general; therefore, its kurtosis value is too larger than zero.

Kurtosis detector is based on kurtosis value of the received signal [7]. In [7] the KD receiver is proposed for PPM signaling scheme. The KD receiver structure is shown in Figure 2.

In this paper we used $\mathrm{KD}$ receiver for OOK signaling in IR-UWB systems. In this case, the kurtosis value of the received signal is calculated in receiver as follows

$$
z_{\mathrm{KD}}=\frac{1}{T_{i}} \int_{0}^{T_{i}} r^{4}(t) \mathrm{d} t-3\left\{\frac{1}{T_{i}} \int_{0}^{T_{i}} r^{2}(t) \mathrm{d} t\right\}^{2},
$$

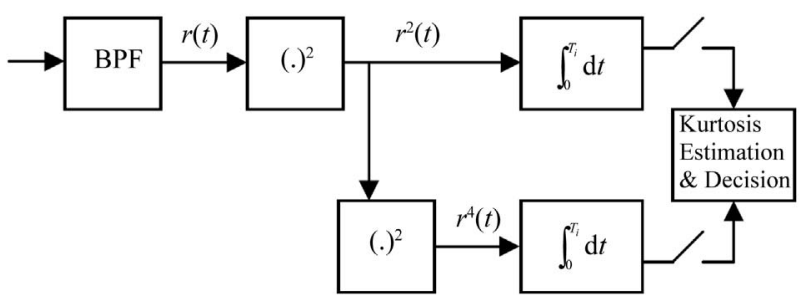

Figure 2. Kurtosis detector (KD) structure proposed in [7]. 
and then, two hypotheses in KD receiver are defined as follows

$$
\left\{\begin{aligned}
H_{K 0}: z_{\mathrm{KD}}= & \frac{1}{T_{i}} \int_{0}^{T_{i}} n^{4}(t) \mathrm{d} t-3\left\{\frac{1}{T_{i}} \int_{0}^{T_{i}} n^{2}(t) \mathrm{d} t\right\}^{2} \\
H_{K 1}: z_{\mathrm{KD}}= & \frac{1}{T_{i}} \int_{0}^{T_{i}}(g(t)+n(t))^{4} \mathrm{~d} t \\
& -3\left\{\frac{1}{T_{i}} \int_{0}^{T_{i}}(g(t)+n(t))^{2} \mathrm{~d} t\right\}^{2}
\end{aligned}\right.
$$

In $\mathrm{KD}$ receiver case similar to $\mathrm{ED}$ receiver, the symbol decision is made by comparing $z_{\mathrm{KD}}$ with a threshold value $T h_{K}$ :

$$
\left\{\begin{array}{l}
\text { if } z_{\mathrm{KD}}<T h_{\mathrm{Kopt}}: H_{K 0} \\
\text { if } z_{\mathrm{KD}} \geq T h_{\mathrm{Kopt}}: H_{K 1}
\end{array}\right.
$$

where the optimum threshold value $T h_{\mathrm{Kopt}}$ is obtained by the solution of $p_{K 0}(x)=p_{K 1}(x)$, and the functions of $p_{K 0}(x)$ and $p_{K 1}(x)$ are the probability density functions (PDF) of $H_{K 0}$ and $H_{K 1}$ respectively.

By using Maximum likelihood (ML) parameter estimation in simulations the PDFs of $p_{K 0}(x)$ and $p_{K 1}(x)$ can be fitted by Generalized Extreme Value (GEV) distribution density function. The GEV distribution function defined as follows

$$
f(x)=\frac{1}{\sigma}\left[1+\xi\left(\frac{x-\mu}{\sigma}\right)\right]^{-1 / \xi-1} \exp \left\{-\left[1+\xi\left(\frac{x-\mu}{\sigma}\right)\right]^{-1 / \xi}\right\}
$$

where $\zeta, \sigma, \mu$ are the parameters of GEV distribution function that obtained from ML parameter estimation.

The semi analytic expression for BER is obtained by using GEV distribution parameters. The parameters of GEV distribution can be obtained from numerical methods in simulations. The approximation of threshold value is obtained by solving the following equation,

$$
\begin{aligned}
& \frac{1}{\sigma_{0}}\left[1+\xi_{0}\left(\frac{T h_{\mathrm{gev}}-\mu_{0}}{\sigma_{0}}\right)\right]^{-1 / \xi_{0}-1} \exp \left\{-\left[1+\xi_{0}\left(\frac{T h_{\mathrm{gev}}-\mu_{0}}{\sigma_{0}}\right)\right]^{-1 / \xi_{0}}\right\} \\
& =\frac{1}{\sigma_{1}}\left[1+\xi_{1}\left(\frac{T h_{\mathrm{gev}}-\mu_{1}}{\sigma_{1}}\right)\right]^{-1 / \xi_{1}-1} \exp \left\{-\left[1+\xi_{1}\left(\frac{T h_{\mathrm{gev}}-\mu_{1}}{\sigma_{1}}\right)\right]^{-1 / \xi_{1}}\right\},
\end{aligned}
$$

where $\left(\zeta_{0}, \sigma_{0}, \mu_{0}\right)$ and $\left(\zeta_{1}, \sigma_{1}, \mu_{1}\right)$ are the parameters of GEV distribution for hypothesis 0 and hypothesis 1 respectively. By using the approximation of threshold value $\left(T h_{\mathrm{gev}}\right)$, the BER expression of bit 0 can be evaluated as

$$
P_{\mathrm{e} \mid 0}=\int_{T h_{\mathrm{gev}}}^{\infty} \frac{1}{\sigma_{0}}\left[1+\xi_{0}\left(\frac{x-\mu_{0}}{\sigma_{0}}\right)\right]^{-1 / \xi_{0}-1} \exp \left\{-\left[1+\xi_{0}\left(\frac{x-\mu_{0}}{\sigma_{0}}\right)\right]^{-1 / \xi_{0}}\right\} \mathrm{d} x=1-\exp \left\{-\left[1+\xi_{0}\left(\frac{T h_{\mathrm{gev}}-\mu_{0}}{\sigma_{0}}\right)\right]^{-1 / \xi_{0}}\right\},
$$

and, the BER expression of bit 1 can be evaluated as

$$
P_{\mathrm{e} \mid 1}=\int_{-\infty}^{T h_{\mathrm{gev}}} \frac{1}{\sigma_{1}}\left[1+\xi_{1}\left(\frac{x-\mu_{1}}{\sigma_{1}}\right)\right]^{-1 / \xi_{1}-1} \exp \left\{-\left[1+\xi_{1}\left(\frac{x-\mu_{1}}{\sigma_{1}}\right)\right]^{-1 / \xi_{1}}\right\} \mathrm{d} x=\exp \left\{-\left[1+\xi_{1}\left(\frac{T h_{\mathrm{gev}}-\mu_{1}}{\sigma_{1}}\right)\right]^{-1 / \xi_{1}}\right\} .
$$

Finally, the BER can be expressed as

$$
P_{\mathrm{e}}=0.5 P_{\mathrm{e} \mid 0}+0.5 P_{\mathrm{e} \mid 1} \text {. }
$$

\subsection{Fourth Power Detector}

In [8], improved ED receiver is proposed by replacing the squaring operation in ED receiver with an arbitrary positive power operation. In this paper the fourth power operation is used for OOK IR-UWB signalling scheme, which has been called fourth power detector (FD). We used FD receiver because it is more practical and it has better performance.

The structure of FD receiver, which is shown in Fig- ure 3, is similar to the ED receiver except that the FD receiver employs two square devices. The decision variable in FD is obtained using the following expression

$$
z_{\mathrm{FD}}=\int_{0}^{T_{i}} r^{4}(t) \mathrm{d} t
$$

and the two hypotheses are defined as follow

$$
\begin{cases}H_{F 0}: z_{\mathrm{FD}}=\int_{0}^{T_{i}} n^{4}(t) \mathrm{d} t & \text { (bit 0) } \\ H_{F 1}: z_{\mathrm{FD}}=\int_{0}^{T_{i}}(g(t)+n(t))^{4} \mathrm{~d} t & \text { (bit } 1)\end{cases}
$$

Similar to ED and KD receivers, the symbol decision in $\mathrm{FD}$ receiver is made by comparing $z_{\mathrm{FD}}$ with a threshold value $T h_{F}$ : 


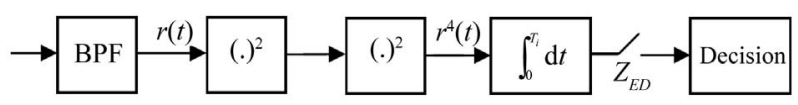

Figure 3. Fourth power detector (FD).

$$
\left\{\begin{array}{cccc}
\text { if } & z_{\mathrm{FD}}<T h_{\mathrm{Fopt}}: & H_{F 0} \\
\text { if } & z_{\mathrm{FD}} \geq T h_{\mathrm{Fopt}}: & H_{F 1}
\end{array},\right.
$$

where the optimum threshold value $T h_{\mathrm{Fopt}}$ is obtained by solving $p_{F 0}(x)=p_{F 1}(x)$. $p_{F 0}(x)$ and $p_{F 1}(x)$ are the probability density functions of $H_{F 0}$ and $H_{F 1}$, respectively.

In [8], the PDFs of $p_{F 0}(x)$ and $p_{F 1}(x)$ are approximated by using Gamma distribution function. In this paper, these PDFs are approximated by GEV distribution function which have the same relations as $p_{K 0}(x)$ and $p_{K 1}(x)$. Results of simulation in section $\mathrm{V}$ show that the GEV distribution function has higher accuracy than Gamma distribution function.

The semi analytic expression of BER for FD receiver can be calculated from Equation (16). In this equation, parameters of GEV distribution for $H_{F 0}$ and $H_{F 1}$ are obtained by ML parameter estimation.

\section{Simulation Results}

Simulations are done in IEEE 802.15.4a CM1 channel model [9] with maximum delay spread ( $\left.T_{\text {mds }}\right)$ truncated to $200 \mathrm{nsec}$. The second derivative of Gaussian pulse is used with pulse duration $T_{p}=1.5 \mathrm{nsec}$, and the symbol duration is $T_{b}=400 \mathrm{nsec}$. The energy of the channel impulse response is normalized to have unit power gain, i.e. $\sum \alpha_{j}^{2}=1$. We also assume perfect synchronization.

Figures 4 and 5 show the accurate cumulative density functions (CDFs) and fitted GEV CDFs for $H_{K 0}$ and $H_{K 1}$. Figures 6 and 7 show the accurate CDFs, fitted GEV, and fitted Gamma CDFs of $H_{F 0}$ and $H_{F 1}$, respectively. The accurate CDFs are obtained by using the histogram method and GEV and Gamma approximate CDFs are obtained by using ML estimation of distribution parameters.

According to the above-mentioned figures, GEV CDFs fitted to $H_{K 0}, H_{K 1}, H_{F 0}$ and $H_{F 1}$ CDFs have high accuracy for different amounts of $E_{b} / N_{0}$ and integration intervals. In FD receiver the fitted GEV and Gamma distributions of $H_{F 0}$ have almost the same accuracy, while in the case of $H_{F 1}$, the fitted GEV CDF has a better accuracy than the fitted Gamma CDF.

Figure 8 shows the bit error rate (BER) performance of $\mathrm{ED}, \mathrm{KD}$ and FD receivers as a function of integration interval $\left(T_{i}\right)$ for sample amounts of $E_{b} / N_{0}=14 \mathrm{~dB}$ and $E_{b} / N_{0}=16 \mathrm{~dB}$. In ED and FD receivers, there is an optimum integration interval that minimizes the BER. Increasing the amount of $E_{b} / N_{0}$ causes this optimum value

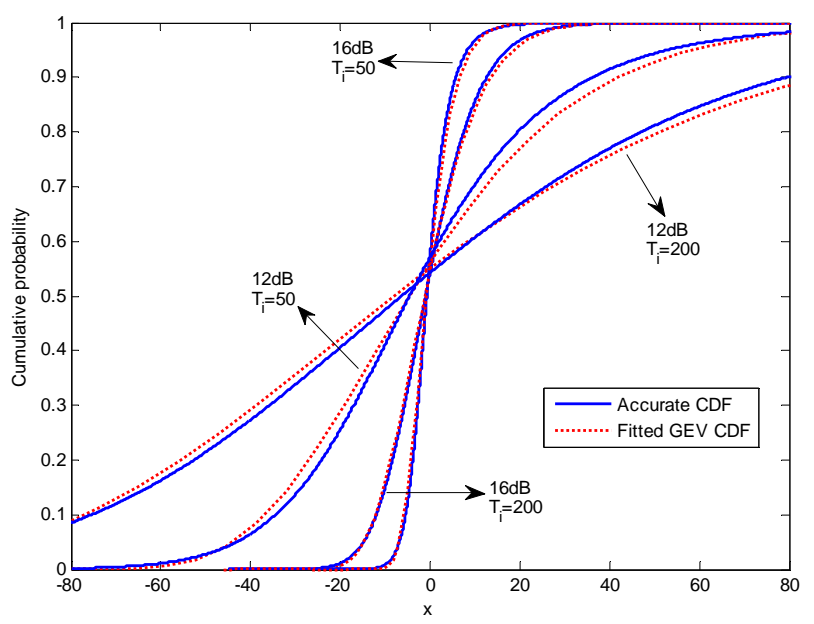

Figure 4. CDFs of hypothesis 0 in $\mathrm{KD}$ receiver $\left(H_{K 0}\right)$ for $T_{i}=$ 50 nsec, 200 nsec and $E_{b} / N_{0}=12 \mathrm{~dB}, 16 \mathrm{~dB}$.

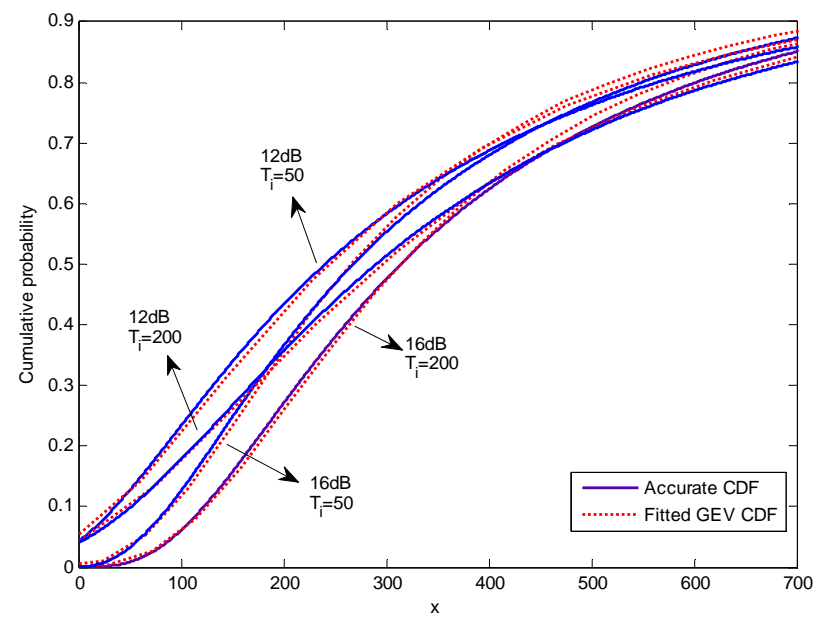

Figure 5. CDFs of hypothesis 1 in $\mathrm{KD}$ receiver $\left(H_{K 1}\right)$ for $T_{i}=$ 50nsec, 200 nsec and $E_{b} / N_{0}=12 \mathrm{~dB}, 16 \mathrm{~dB}$.

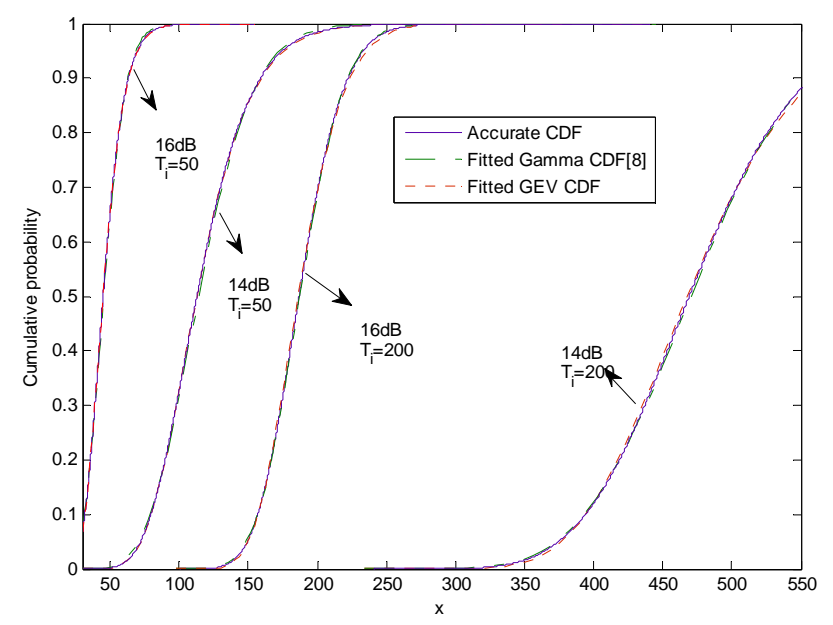

Figure 6. CDFs of hypothesis 0 in FD receiver $\left(H_{F 0}\right)$ for $T_{i}=$ 50 nsec, 200 nsec and $E_{b} / N_{0}=14 \mathrm{~dB}, 16 \mathrm{~dB}$. 


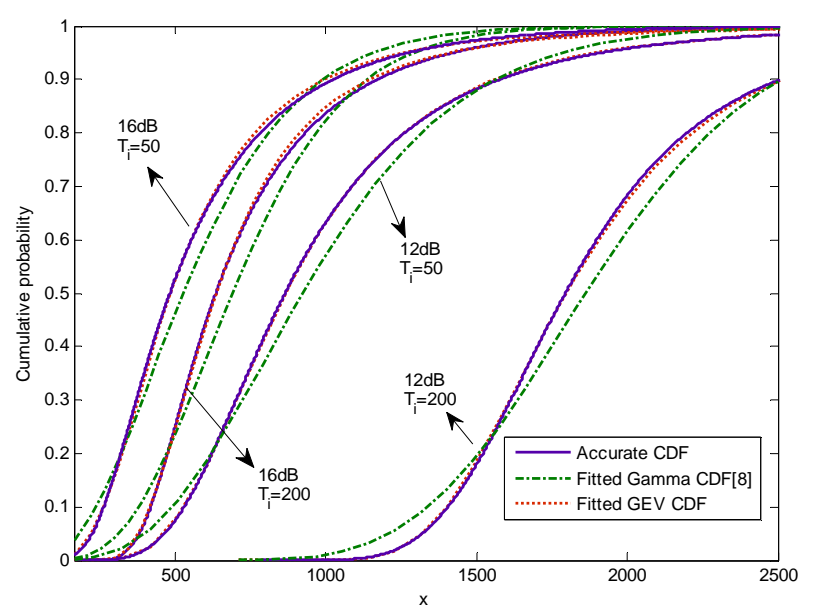

Figure 7. CDFs of hypothesis 1 in FD receiver $\left(H_{F 1}\right)$ for $T_{i}=$ 50 nsec, 200 nsec and $E_{b} / N_{0}=12 \mathrm{~dB}, 16 \mathrm{~dB}$.

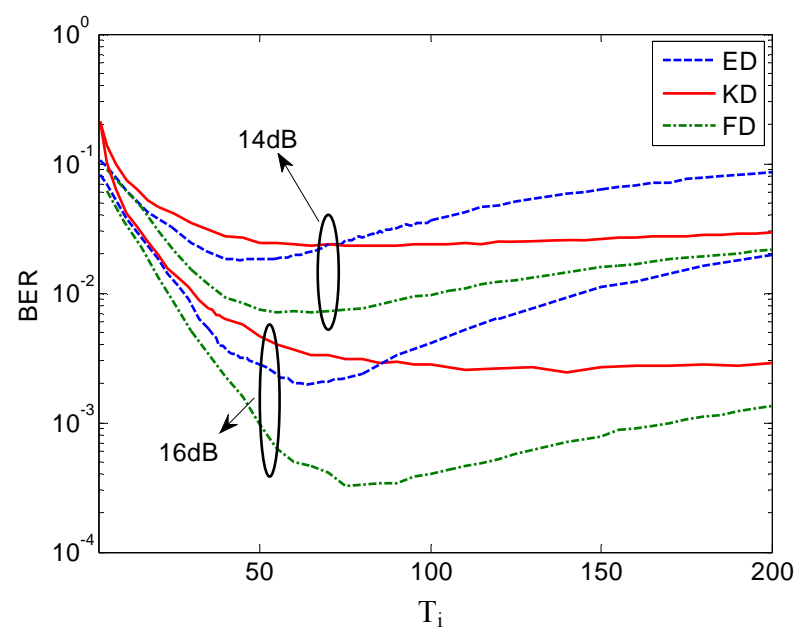

Figure 8. BER vs integration interval $\left(T_{\mathrm{mds}}=200 \mathrm{nsec}\right)$.

to increase. In $\mathrm{KD}$ receiver, short integration intervals have a negative effect on the performance, and BER is almost constant for large values of integration intervals. For large integration intervals, the KD receiver does not require optimization of integration interval. The FD receiver outperforms $\mathrm{ED}$ and $\mathrm{KD}$ receivers for almost all amounts of integration intervals.

Figure 9 shows the BER performance of ED, FD and KD receivers for integration interval $T_{i}=T_{\mathrm{mds}}=200 \mathrm{nsec}$. This figure also demonstrates the BER of ED and FD for optimum integration intervals. For $T_{i}=200 \mathrm{nsec}$ and $\mathrm{BER}=10^{-3}$, the $\mathrm{KD}$ receiver has a $1.4 \mathrm{~dB}$ better performance than the $\mathrm{ED}$ receiver and the $\mathrm{FD}$ receiver has a $2 \mathrm{~dB}$ better performance than the ED receiver. The ED receiver with optimized integration interval has a $0.2 \mathrm{~dB}$ better performance than the KD receiver for high values of $E_{b} / N_{0}$. The FD receiver with the optimum integration intervals has a better performance than $\mathrm{ED}$ and $\mathrm{KD}$ receivers in all values of $E_{b} / N_{0}$. For BER $=10^{-4}$, the FD

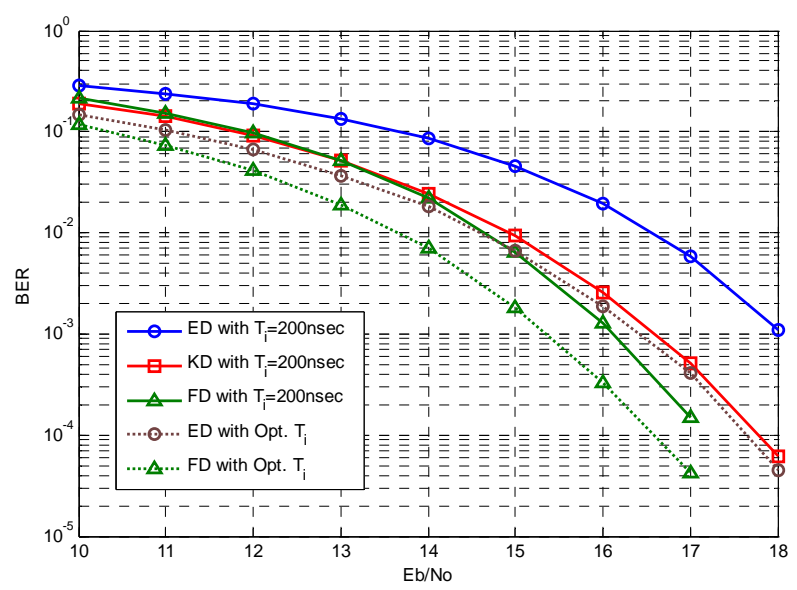

Figure 9. BER comparison of ED, FD and KD receivers.

receiver with optimized integration interval has a $1 \mathrm{~dB}$ better performance than the ED receiver with optimized integration interval and $1.2 \mathrm{~dB}$ better performance than the $\mathrm{KD}$ receiver.

Since the integration interval depends on maximum delay spread of channel and SNR value, using the optimum integration intervals in the practical systems is difficult. Considering that for large integration intervals KD receiver does not require optimization of integration interval, therefore the $\mathrm{KD}$ receiver with large integration interval can be used for practical systems.

Figure 10 shows the BER performance of KD and FD receivers for integration interval of $50 \mathrm{nsec}$. In $\mathrm{KD}$ receiver, the BER using GEV approximation of threshold has a high accuracy for almost all amounts of $E_{b} / N_{0}$, and for high values of $E_{b} / N_{0}$, the BER using GEV approximation of threshold has a performance about $0.2 \mathrm{~dB}$ worse than the BER with optimum threshold. The semi analytic BER of KD receiver has great accuracy for medium and low $E_{b} / N_{0}$, and for high values of $E_{b} / N_{0}$ is 0.6 $\mathrm{dB}$ different from the BER simulation result. In FD receiver the BER using GEV approximation of threshold is highly accurate for all amounts of $E_{b} / N_{0}$, and the semi analytic BER of FD receiver has a high accuracy for medium and low $E_{b} / N_{0}$, and for high values of $E_{b} / N_{0}$ is 0.5 $\mathrm{dB}$ different from BER simulation result.

The BER using optimum threshold and GEV approximation of threshold and the semi analytic BER of $\mathrm{KD}$ and FD receivers for integration interval of $200 \mathrm{nsec}$ are shown in Figure 11. Similar to $T_{i}=50 \mathrm{nsec}$, for $T_{i}=$ $200 \mathrm{nsec}$ the BER using GEV approximation of threshold has high accuracy for all amounts of $E_{b} / N_{0}$ for KD and FD receivers. For BER $=10^{-3}$, the semi analytic BER is $0.5 \mathrm{~dB}$ different from BER simulation result for $\mathrm{KD}$ and $0.3 \mathrm{~dB}$ different from BER simulation results for FD.

The results of Figures $\mathbf{1 0}$ and $\mathbf{1 1}$ show that the semi analytic BER and simulation BER have different values in high amounts of $E_{b} / N_{0}$. This is because the GEV 


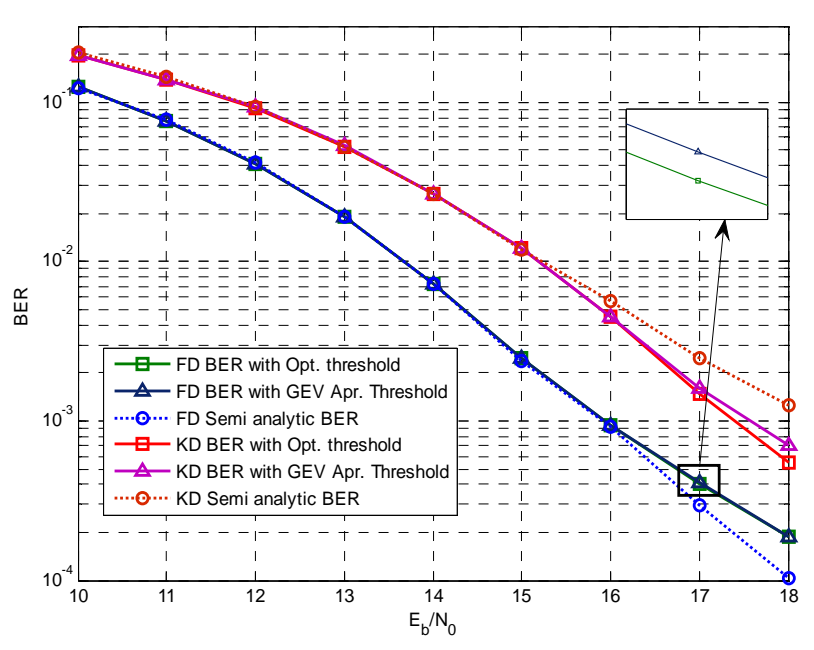

Figure 10. BER performances of $\mathrm{KD}$ and $\mathrm{FD}$ receivers with GEV approximation of threshold and semi analytic method in $T_{i}=50$ nsec.

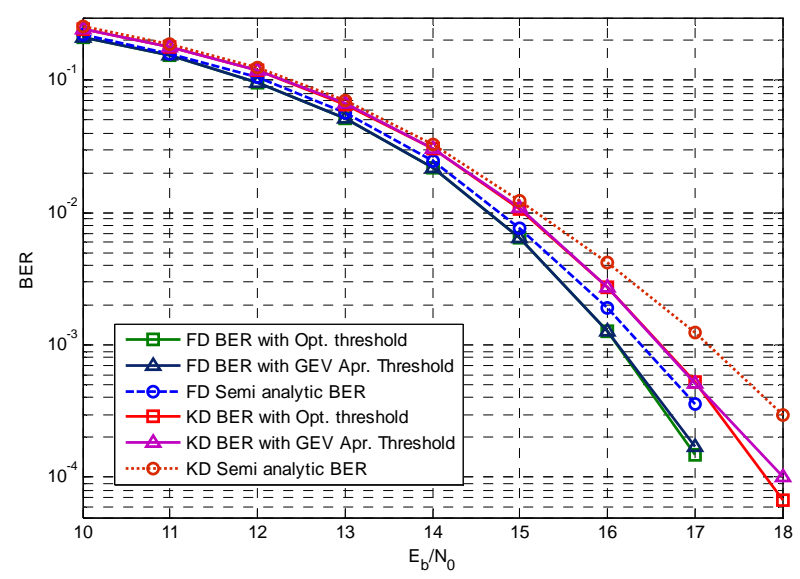

Figure 11. BER performances of $K D$ and $F D$ receivers with GEV approximation of threshold and semi analytic method in $T_{i}=200$ nsec.

function has not high accuracy for tails of hypo- theses PDFs and the BER in high values of $E_{b} / N_{0}$ depends on tails of hypotheses PDFs.

In Figures 12 and 13, absolute variations of BER normalized to BER in optimum threshold versus the percent of optimum threshold variations are illustrated for sample amounts of $E_{b} / N_{0}=14 \mathrm{~dB}$ and $E_{b} / N_{0}=16 \mathrm{~dB}$. Sensitivity of $\mathrm{KD}$ receiver to the optimum threshold variations is considerably lower than $\mathrm{ED}$ and $\mathrm{KD}$ receivers. This is because in KD the PDFs of $H_{K 0}$ and $H_{K 1}$ have low slopes in optimum threshold point (Figure 14). Therefore by changing optimum threshold value, the BER is not greatly increased. But in ED and FD receivers, the PDFs of hypotheses 0 and 1 have high slopes in the optimum threshold point (Figures 15 and 16); therefore small changes in the optimum threshold value cause a great increase in the BER.

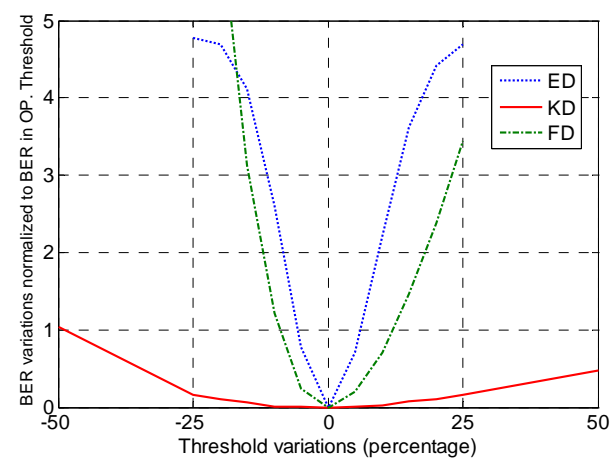

Figure 12. Absolute variations of BER normalized to BER in optimum threshold vs. optimum threshold variations (percentage) for $E_{b} / N_{0}=14 \mathrm{~dB}$ and $T_{i}=200$ nsec.

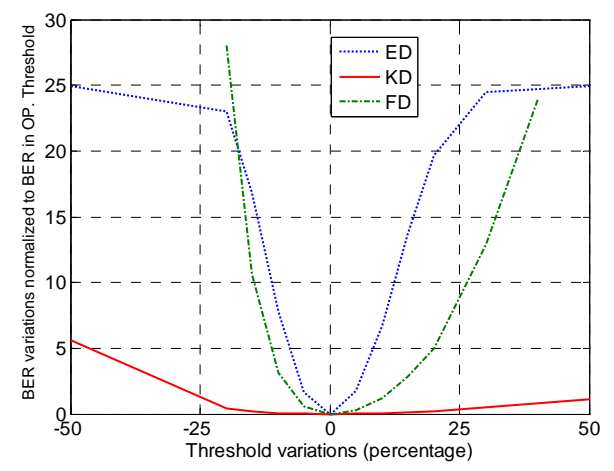

Figure 13. Absolute variations of BER normalized to BER in optimum threshold vs. optimum threshold variations (percentage) for $E_{b} / N_{0}=16 \mathrm{~dB}$ and $T_{i}=200$ nsec.

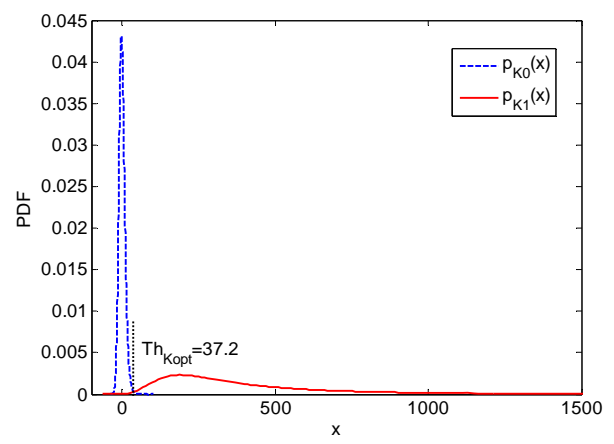

Figure 14. PDFs of two hypotheses in $\mathrm{KD}\left(E_{b} / N_{0}=16 \mathrm{~dB}\right)$.

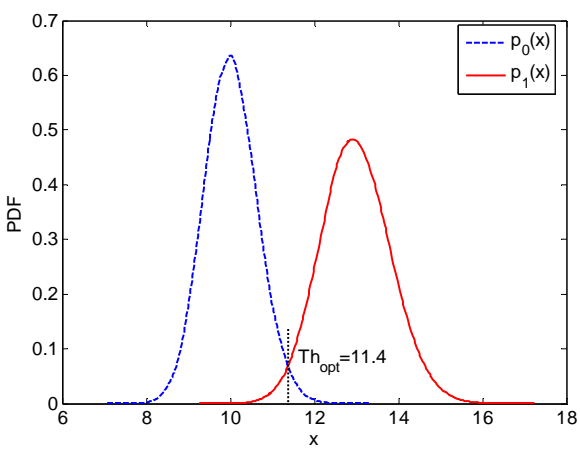

Figure 15. PDFs of two hypotheses in $\operatorname{ED}\left(E_{b} / N_{0}=16 \mathrm{~dB}\right)$. 


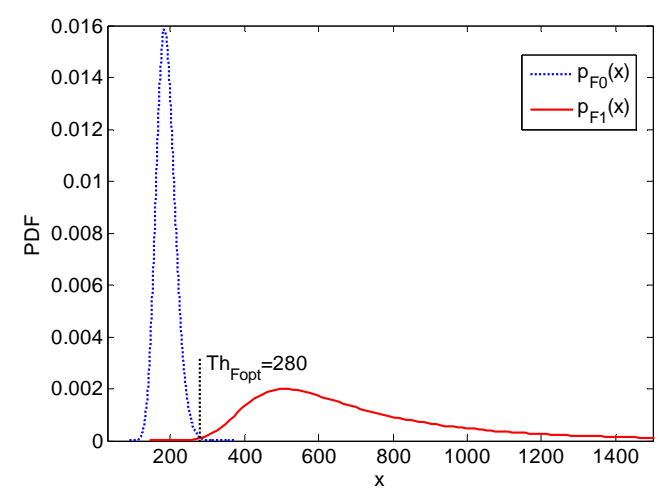

Figure 16. PDFs of two hypotheses in FD $\left(E_{b} / N_{0}=16 \mathrm{~dB}\right)$.

Since practical UWB systems are time varying and SNR values in these systems are variable, the optimum threshold value has variations. That's why the KD receiver can be used in practical UWB systems due to its better robustness to optimum threshold variations than $\mathrm{ED}$ and FD receivers.

In this paper, we assume that the maximum delay spread of channel is truncated to $200 \mathrm{nsec}$. For avoiding inter symbol interference, the symbol interval must be larger than $200 \mathrm{nsec}$. For all amounts of symbol interval that is greater than $200 \mathrm{nsec}$, the results of this paper do not change. Simulation results in [7] show that KD receiver has a better performance for low values of maximum delay spread.

\section{Conclusion}

In this paper, the $\mathrm{KD}$ and $\mathrm{FD}$ receivers were proposed for OOK modulation scheme. The FD receiver has a better performance than $\mathrm{ED}$ and $\mathrm{KD}$ receivers in almost all values of integration intervals. For high integration intervals the $\mathrm{KD}$ receiver outperforms $\mathrm{ED}$ receiver about 2 $\mathrm{dB}$ for low values of $E_{b} / N_{0}$ and $1.4 \mathrm{~dB}$ for high values of $E_{b} / N_{0}$. For high values of $E_{b} / N_{0}$ the ED receiver using optimized integration interval outperforms $\mathrm{KD}$ receiver around $0.2 \mathrm{~dB}$. The GEV distribution function is a good approximation for PDFs of hypotheses 0 and 1 in IR-UWB OOK scheme with KD and FD receivers. The approximation of optimum threshold value and the semi analytic BER expression can be calculated from GEV distribution function. The KD receiver does not require optimization of integration interval, and the sensitivity to the optimum threshold value in KD receiver is very low compared to ED and FD receivers. Therefore, KD receiver can be the better option for practical purposes.

\section{Acknowledgements}

The authors would like to thank the Iranian Institute of information and Communication Technology (the former ITRC) for the support of this work.

\section{REFERENCES}

[1] M. Z. Win and R. A. Scholtz, "Impulse Radio: How It Works," IEEE Communication Letter, Vol. 2, No. 2, 1998, pp. 36-38. doi:10.1109/4234.660796

[2] L. Yang and G. Giannakis, "Ultra-Wideband Communications: An Idea Whose Time Has Come," IEEE Signal Processing Magazine, Vol. 21, No. 6, 2004, pp. 26-54. doi:10.1109/MSP.2004.1359140

[3] K. Witrisal, G. Leus, G. Janssen, M. Pausini, F. Troesch, T. Zasowski and J. Romme, "Noncoherent Ultra-Wideband Systems," IEEE Signal Processing Magazine, Vol. 26, No. 4, 2009, pp. 48-66. doi:10.1109/MSP.2009.932617

[4] P. A. Humblet and M. Azizoglu, "On the Bit-Error Rate of Lightwave Systems with Optical Amplifiers," Journal of Lightwave Technology, Vol. 9, 1991, pp. 1576-1582.

[5] M. E. Sahin, I. Guvenc and H. Arslan, "Optimization of Energy Detector Receivers for UWB Systems," Proceedings of IEEE Vehicular Technology Conference, Dallas, Vol. 2, 30 May-1 June 2005, pp. 1386-1390.

[6] X. T. Cheng, Y. L. Guan and Y. Gong, "Thresholdless Energy Detection for Ultra-Wideband Block-Coded OOK Signals" Electronics Letters, Vol. 44, No. 12, 2008, p. 755. doi:10.1049/el:20083565

[7] M. Khan, B. Sallberg, G. Nordberg and I. Claesson, "Noncoherent Detection of Impulse Radio UWB Signal Based on Fourth Order Statistics," Proceedings of IEEE International Conference on Ultra-Wideband, Vancouver, 911 September 2009, pp. 824-828.

[8] Y. F. Chen, "Improved Energy Detector for Random Signals in Gaussian Noise," IEEE Transaction on Wireless Communication, Vol. 9, No. 2, 2010, pp. 558-563. doi:10.1109/TWC.2010.5403535

[9] A. F. Molisch, K. Balakrishnan, D. Cassioli, C.-C. Chong, S. Emami, A. Fort, J. Karedal, J. Kunisch, H. Schantz, U. Schuster and K. Siwiak, "Channel Model-Final Report," IEEE 802.15.4a, 2004. 